\title{
Health provider experiences with galactagogues to support breastfeeding: a cross-sectional survey
}

\author{
Alessandra N Bazzano' \\ Lisa Littrell' \\ Amelia Brandt ${ }^{\prime}$ \\ Shelley Thibeau ${ }^{2}$ \\ Kamala Thriemer ${ }^{3}$ \\ Katherine P Theall' \\ 'Department of Global Community \\ Health and Behavioral Sciences, Tulane \\ University School of Public Health and \\ Tropical Medicine, ${ }^{2}$ Ochsner Health \\ System, New Orleans, LA, USA; ${ }^{3}$ MLT \\ EpiConsult, Jingili, NT, Australia
}

This article was published in the following Dove Press journal:

Journal of Multidisciplinary Healthcare

17 November 2016

Number of times this article has been viewed

Background: Exclusive breastfeeding for infants up to 6 months is widely recommended, yet breastfeeding rates are relatively low in the US. The most common reason women stop breastfeeding early is a perceived insufficiency of milk. Galactagogues are herbal and pharmaceutical products that can help increase milk supply; however, data on their efficacy and safety is limited. Lactation consultants, obstetricians, and other health providers are an important point of contact for breastfeeding women experiencing challenges with lactation. This study explored providers' perceptions, experiences, and practices in relation to galactagogue recommendation. Method: A cross-sectional survey was conducted among a convenience sample of Englishspeaking health providers in the US who counsel breastfeeding women and their infants.

Results: More than $70 \%$ of respondents reported to recommend galactagogues. The most frequently recommended galactagogue was fenugreek with respondents indicating that they recommend it either 'always' (8.5\%) or 'most of the time' $(14.9 \%)$ and 'sometimes' $(46.8 \%)$. More than $80 \%$ of the respondents indicated that galactagogues were useful for their clients and only one-third reported side effects. Reasons for refraining from recommending galactagogues were insufficient evidence of its efficacy and safety. Respondents reported a wide variety of sources of information used for their own education about galactagogues.

Discussion: Despite little evidence regarding safety and efficacy, some galactagogues are widely recommended and often perceived to be useful. However, concerns about their efficacy and safety remain. In order to assure both providers and users about safety and efficacy, more robust studies as well as better pharmacovigilance systems are needed.

Keywords: lactation, human milk, nutrition, clinical decision making, lactogenesis

\section{Introduction}

Exclusive breastfeeding for infants until the age of 6 months, with continued breastfeeding to a minimum of 2 years, is strongly endorsed by the World Health Organization, and numerous advocacy efforts have followed this recommendation globally. ${ }^{1,2}$ Recommendations by the American Academy of Pediatrics and other organizations in the US also emphasize the importance of this practice due to the health benefits for both mother and infant, and the recognition of breastfeeding as the normative mode of infant feeding. ${ }^{3,4}$ Over the last few decades, breastfeeding has been shown to reduce the risk of acute otitis media, nonspecific gastroenteritis, atopic dermatitis, and sudden unexpected infant death. ${ }^{5,6}$ However, breastfeeding rates remain far too low in the US, and one out of every two mothers stops breastfeeding earlier than recommended. ${ }^{7,8}$

Correspondence: Alessandra N Bazzano Department of Global Community Health and Behavioral Sciences, Tulane University School of Public Health and Tropical Medicine, I 440 Canal St, New Orleans, LA 70112, USA

Tel +l 5049882338

Email abazzano@tulane.edu 
Clinicians, such as lactation consultants, physicians, and nurses, are typically the most important point of contact for breastfeeding women experiencing challenges with lactation. They rely on their training and education on the normal process of lactation ${ }^{5}$ in providing valuable guidance and support. ${ }^{9}$ Evidence around various clinical support approaches for lactation is not extensive, but indicates that counseling methods perform well. ${ }^{10}$ While primary care facilities are one venue for such counseling, ${ }^{11}$ obstetric and/or pediatric practices are likely the more common points of contact for lactation counseling.

A recent systematic review of breastfeeding promotion interventions found that education around breastfeeding and/ or supportive measures increased exclusive breastfeeding rates. Interestingly, a combination of group counseling with individual support was superior to each of the measures alone, possibly indicating that peer information-sharing is important. ${ }^{12}$ Another recent review indicated that support given at an early postnatal stage and with a duration over extended periods of time had a positive impact; however, this review was limited by the quality of the included studies. ${ }^{15}$ None of the studies included in the review assessed adherence to the intervention, and only two studies included important factors such as maternal body mass index, which is known to impact prolactin levels and lactation. ${ }^{13}$

The most commonly reported reason for mothers to prematurely end breastfeeding is the perception that milk quantity is insufficient to address the needs of the infant. ${ }^{8}$ This perception among lactating women has been confirmed in a variety of studies internationally., ${ }^{7,8}$ There are multiple reasons for reduced maternal milk supply, both preventable and biological, including suboptimal breastfeeding technique, deficient glandular tissue, and hormonal imbalance. ${ }^{14}$ Once the latter two reasons are ruled out, lactation counseling and education about improving breastfeeding techniques should commence. If this is not successful, galactagogues may be considered by health care workers as an additional supportive measure.

Galactagogues are a group of substances, drugs and herbal remedies, used to help increase lactation or secretion of breast milk. The most commonly reported to be used are dopamine D2 receptor antagonists such as Reglan (metoclopramide) and Motilium (domperidone). Herbal remedies used as galactagogues include fenugreek, blessed thistle, milk thistle, goat's rue, and fennel. ${ }^{15}$ However, there is an important dearth of evidence regarding efficacy and the safety of these substances. There is little data available from randomized studies on the off-label use of domperidone ${ }^{16,17}$ and metoclopramide, ${ }^{18-21}$ and existing studies are limited by either small sample sizes or inconclusive results. Similarly, there is a lack of robust information on herbal substances. Although herbal remedies are often viewed as harmless or safe, few regulatory guidelines exist to ensure safety and efficacy of these substances. Unlike registered drugs, herbal remedies can be sold without evidence of their clinical usefulness or the absence of potentially harmful effects.

Studies among lactating women using galactagogues revealed that most women using these substances perceived them to be effective, with fenugreek and blessed thistle being used most frequently, and often in combination. ${ }^{22-25}$ Furthermore, women also expressed the notion that herbal galactagogues are safer compared to conventional medicine and expected providers to be aware of the different options and hence able to provide adequate advice. ${ }^{26}$ Providers face insufficient clinical guidance and a lack of protocols on galactagogue use, and recently this gap in reliable data has led to more cautious recommendation about galactagogues use. ${ }^{23}$ Nevertheless, health providers counseling lactating women are likely to have experience with patients' use of galactagogues and are frequently requested to provide recommendations to patients when asked. In order to understand the experiences, perceptions, and practice of health providers who may have patients using galactagogues, a cross-sectional survey delivered online to health care providers in the US was conducted.

\section{Methods}

\section{Participants and setting}

A cross-sectional survey of 82 participants was carried out between December 2014 and January 2016 with a convenience sample of English-speaking health providers, including lactation counselors, pediatricians, doulas, and obstetrician/gynecologists, who provide care to breastfeeding women and their infants. Potential participants were initially identified through health clinics and hospitals located near the author's research institutions, and were subsequently emailed an invitation to participate. Respondents were also encouraged to share the survey invitation with any interested colleagues, without restriction on location. All participants provided informed consent (electronically; indicated by clicking to complete the survey) after reading the study information, which was included in the email invitation. No financial or other incentives were offered to participants for completion of the survey. The study was reviewed by the Tulane University Institutional Review Board and the Ochsner Health System Institutional Review Board. The boards deemed the study exempt from further oversight as it was determined to be research involving survey or interview procedures with adult subjects, and meets the criteria of 45 CFR 46.101(b)(2) (US Department of Health and Human Services) to be exempt research. 


\section{Data collection}

The anonymous, 27-item survey was developed and delivered through the Health Insurance Portability and Accountability Act compliant Qualtrics platform and all transmitted data was protected by Transport Layer Security encryption. Respondents' contact information was stored separately from survey responses to maintain anonymity. The research team reviewed the survey multiple times prior to release, to ensure flow and readability, and it was pretested with a small sample of providers.

The survey contained 19 questions with fixed responses and eight open-ended questions. The survey took approximately 10 minutes to complete. Participant responses were categorized as follows.

\section{Demographic and professional characteristics (five items)}

Participants were asked to provide information on gender, ethnicity, race, professional specialty, and length of practice in their career. Four of the questions were fixed-response, while length of practice was open-ended.

\section{Galactagogue prescription practices (16 items)}

This section utilized a skip pattern that differentiated responses from those who had prescribed a galactagogue from those who had never done so. Respondents were asked a fixed-response question regarding whether or not they had ever prescribed or recommended any product to increase a mother's milk supply. Those who answered 'yes' were asked nine additional fixed-response questions regarding prescription frequency for specific galactagogues. These questions used a five-point Likert-scale ${ }^{27}$ ranging from 'never' to 'always'. Questions in this section also gathered information regarding typical time frame and dosage for recommending, while another asked about where providers seek information on galactagogues. Respondents who had never prescribed or recommended any product were asked an open-ended question regarding their reasons for not having done so. Concerns over recommending galactagogues was assessed by a question with dichotomous response. Those who responded affirmatively were asked an open-ended question requesting more information about their concerns.

\section{Perception of galactagogues (six items)}

This section included fixed-answer items asking about whether providers perceive that galactagogues increased milk supply for patients when prescribed/recommended and whether the provider felt that galactagogues were useful for their patient. Additional questions asked respondents about whether any patient had ever experienced side effects or adverse effects. Each item also included an open-ended follow-up question asking the respondent to list the side effects or adverse effects if the respondent answered affirmatively.

\section{Data analysis}

SPSS Statistical Package for Windows, version 23 (IBM Corporation, Armonk, NY, USA) was used for data cleaning and analysis. Descriptive analysis of all the study variables was conducted. Counts and frequencies were calculated for the variables which had Likert scale response. The variable 'length of practice' was recoded from a continuous to a categorical variable for reporting purposes; all other variables were kept in original form. Any unanswered questions that were not a result of the survey's skip pattern were treated as missing values and not included in the analysis. Graphical presentation of the results was generated through the use of Microsoft Excel (version 16.0.7167.2040; Microsoft Corporation, Redmond, WA, USA).

\section{Results}

\section{Sample characteristics}

A total of 82 providers were asked to participate in the survey and 71 completed and submitted the survey form. The vast majority of providers who responded to the survey were females (95.3\%), while male providers made up only $4.7 \%$ of respondents. The most common type of providers participating in the survey were lactation consultants, comprising $25.5 \%$ of respondents. Obstetrician/gynecologists, doulas, and certified nurse-midwives were represented at similar proportions $(14.9 \%)$ ). A smaller number of respondents were pediatricians (10.6\%) and breastfeeding counselors (4.3\%).

There was a wide variation in the amount of work experience among respondents, with nearly half (45.9\%) being in practice for less than 5 years, $37.8 \%$ practicing for 5-15 years, $13.5 \%$ for $16-25$ years, and $2.7 \%$ for over 25 years. Providers predominantly identified as White $(83.7 \%$,), while $9.3 \%$ identified as Black or African-American, 2.3\% as mixed, and $2.3 \%$ as multiracial (Table 1 ).

\section{Recommendation practices}

The majority (70.4\%) of study participants recommended at least one type of galactagogue to patients. Respondents who answered positively to recommending a galactagogue were then asked to rate the frequency with which they prescribed specific types. Frequency of prescribing specific galactagogue types is summarized in Figure 1. 
Table I Provider characteristics (total sample size $=7$ I)

\begin{tabular}{ll}
\hline & $\mathbf{N}(\%)$ \\
\hline Gender (n=43) & \\
Female & $4 \mathrm{I}(95.3)$ \\
Specialty (n=47) & \\
Obstetrician/gynecologist & $7(14.9)$ \\
Pediatrician & $5(10.6)$ \\
Lactation consultant & $12(25.5)$ \\
Doula & $7(14.9)$ \\
Breastfeeding counselor & $2(4.3)$ \\
Certified nurse-midwife & $7(14.9)$ \\
Labor and delivery nurse & $\mathrm{I}(2.1)$ \\
Obstetrics registered nurse & $\mathrm{I}(2.1)$ \\
La Leche League leader & \\
Did not specify & $\mathrm{I}(2.1)$ \\
Years in practice (n=37) & $4(8.5)$ \\
$<5$ & \\
5-15 & $17(45.9)$ \\
16-25 & $\mathrm{I}(37.8)$ \\
$>25$ & $5(13.5)$ \\
Race (n=43) & $\mathrm{I}(2.7)$ \\
Black or African-American & \\
White & $4(9.3)$ \\
Mixed & $36(83.7)$ \\
Multiracial: Caucasian & $\mathrm{I}(2.3)$ \\
Asian & $\mathrm{I}(2.3)$ \\
Ethnicity (n=44) & $\mathrm{I}(2.3)$ \\
Not Hispanic or Latino & \\
\hline Note:La Leche Legue is a nonroftora & $43(97.7)$ \\
\hline
\end{tabular}

Note: ${ }^{a}$ La Leche League is a nonprofit organization that distributes information on, and promotes, breastfeeding.

Specific herbal galactagogues that were mentioned as being recommended 'always' by a proportion of providers included: fenugreek (8.5\%), fennel (2.1\%), milk thistle (2.1\%), and More Milk or More Milk Plus (Motherlove ${ }^{\circledR}$ ) $(2.1 \%)$.
Fenugreek was the galactagogue most recommended by all types of providers and $8.5 \%$ of respondents indicated that they recommend it 'always', while $14.9 \%$ indicated they prescribe it 'most of the time', and $46.8 \%$ 'sometimes'. Respondents reported that metoclopramide and domperidone were 'sometimes' ( $23.4 \%$ and $12.8 \%$ respectively) or 'rarely' (31.9\% and $27.7 \%$ respectively) recommended. For all other galactagogues, the majority of respondents indicated that they would 'rarely' or 'never' recommend them, as illustrated in Figure 1 ( $72.3 \%$ and $6.4 \%$ for fennel; $76.6 \%$ and $8.5 \%$ for goat's rue; $55.3 \%$ and $21.3 \%$ for milk thistle; $67.4 \%$ and $6.5 \%$ for More Milk or More Milk Plus).

\section{Typical dosage and source for dosage information}

More than half of the respondents (55.3\%) indicated that they recommend the use of galactagogues 'as needed' (see Figure 2) regarding dosage. Respondents who chose 'other', described their recommended dosage as dependent on the specific situation of the patient, referred to specific doses they had used in the past, or acknowledged not knowing what a correct dosing schedule should be, as specific protocols or guidelines are unavailable.

Respondents were also given the opportunity to answer an open-ended question about their source of information regarding dosing. Overall, respondents reported a variety of different informational sources including Internet sources, trainings or conferences, literature and other related readings, specialty groups such as the La Leche League or the International Board of Lactation Consultant Examiners,

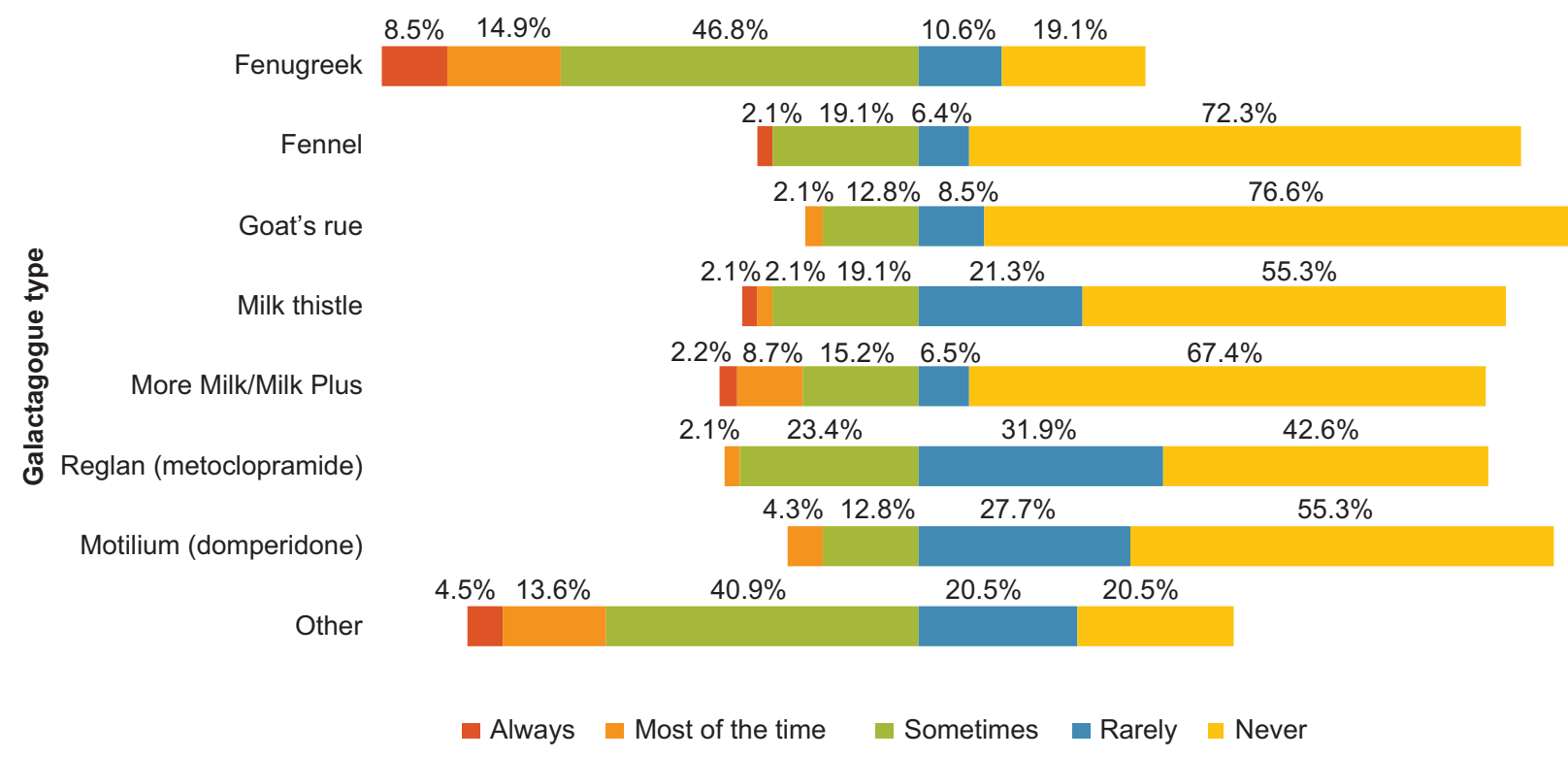

Figure I Frequency of providers recommendation, by galactagogue $(n=47)$. 


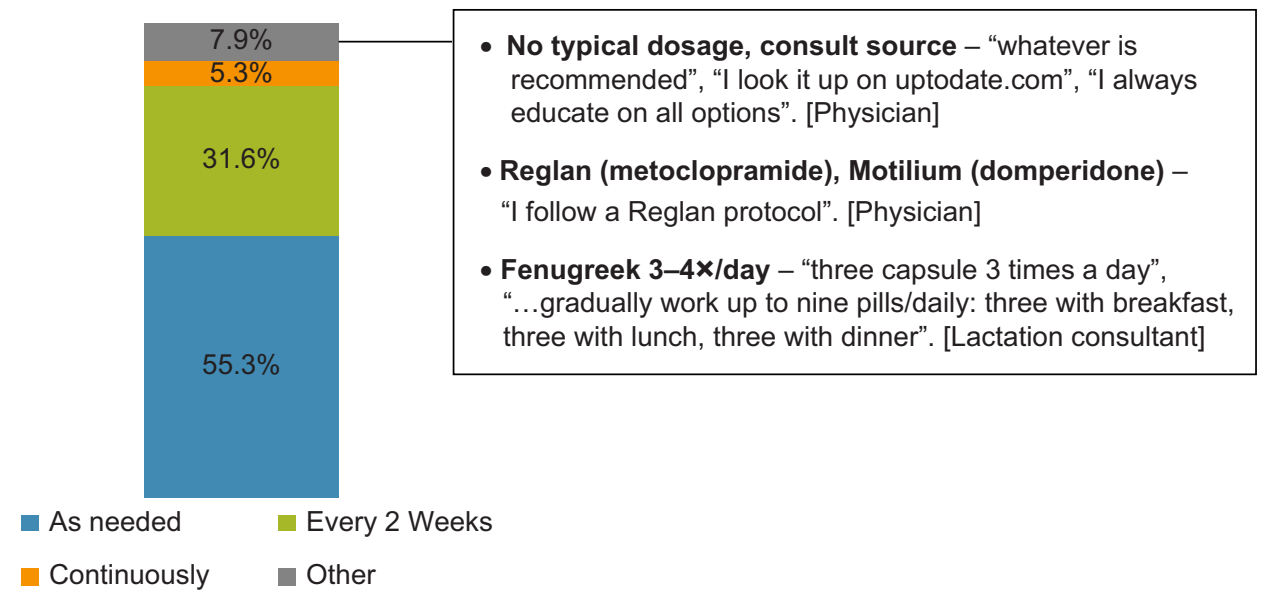

Figure 2 Dosing practices of providers who prescribe or recommend galactagogues.

Table 2 Selected sources of information reported for dosing of galactagogues

- Colleagues: "Many places. Midwifery college, independent research, experience, talking with colleagues". [Midwife]

- Personal experience: respondent was a lactation consultant.

- UpToDate.com and other Internet sources: “Google Search: galactagogue, Dr. Jack Newman, Reglan protocol, domperidone protocol". [Physcian]. "Product websites followed by IBCLE authors' books, articles, and conferences". [Lactation consultant]

- Readings/literature: "Herbalist, literature". [Lactation consultant]. "Reading. Word of mouth from other providers on "proof that it works, and if moms have any increase in milk supply that cannot otherwise be explained". [Lactation consultant]. "Literature research, Dr. Hale's Medication and Mother's Milk handbook, as well as various lactation conferences". [Lactation consultant]

- La Leche Leaguea/IBLCE: all respondents were lactation consultants.

Note: a La Leche League is a nonprofit organization that distributes information on, and promotes, breastfeeding.

Abbrevation: IBLCE, International Board of Lactation Consultant Examiners.

which certifies lactation consultants, and other more informal sources such as colleagues and personal experience (Table 2).

\section{Reasons for not recommending galactagogues}

All respondents regardless of their galactagogue recommendation were asked if they had concerns with recommending galactagogues, and if they answered positively, an open-ended question about those concerns followed. In all, $24.2 \%$ respondents indicated they had concerns. Those who never prescribed or recommended a galactagogue were asked for their reasons and concerns.

Answers to open-ended responses on concerns varied. One obstetrician stated, "I am not familiar with these products. I would consider recommending them if I knew more information on the subject."
Regarding lack of evidence, one physician stated, "Nothing is proven effective - I recommend supportive care and for the mother to decrease stress, bond with baby, eat well, etc...". Another lactation counselor simply responded, "Scientific evidence: is there any?". And another obstetrician stated,

"I have not read or seen any evidence-based information stating that mothers need anything other than regular breastfeeding/the body's natural negative feedback system in order to stimulate milk letdown or increase milk supply".

Regarding the safety, efficacy, and regulation of galactagogues, a midwife who has been in practice for 3 years stated, "Reglan (metoclopramide) has serious side effects and is less effective than domperidone, which can be obtained through pharmacies outside the US. The dangerous side effects of domperidone (which led to its ban in our country [the US]) were associated with intravenous use in cardiac patients, which is largely irrelevant for the population I work with. Neither is perfect. Both have side effects. It's an issue of pick your poison if mothers want to go the pharmaceutical route". A lactation consultant with 30 years' experience stated "Not fully evidence-based; not sure IBCLC [certified lactation consultant] can recommend; not able to follow-up to assess true results".

One provider stated "Galactagogues are interventions into the natural process, but often aren't respected as such. Appropriate interventions obviously improve outcomes and satisfaction, but interventions such as galactagogues are often misused and over-recommended. This can lead people to ignore the source of the problem, which is very often something other than the mother's hormonal milk-production mechanisms." [Midwife, 3 years in practice] 


\section{Commonly reported side effects}

- Depression (Reglan [metoclopramide])

- Gastric distress/nausea (fenugreek)

- Maple syrup smell/body odor (fenugreek)

- Fatigue

- Flushing

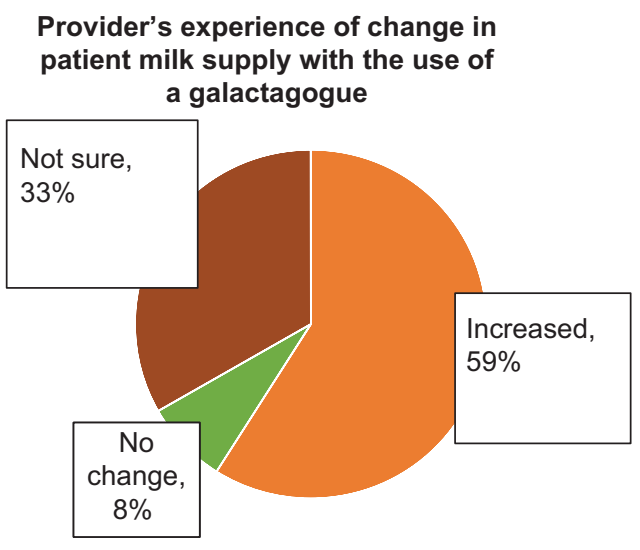

Figure 3 Provider report of perceived usefulness and reported side effects.

\section{Perception of effectiveness and safety}

A large majority of providers $(82.1 \%)$ felt that the galactagogues they had recommended were useful, and an even larger proportion (86.4\%) said that they would recommend a galactagogue again. However, $12.8 \%$ of providers did not feel that galactagogues were useful, and one respondent said that they would not recommend a galactagogue again. Providers indicated that recommending a galactagogue to a typical client resulted in increased milk supply in most cases (60.5\%), while $31.6 \%$ of providers responded that they were not sure if there was a change, and $7.9 \%$ of providers responded that there was no change in milk supply.

Thirty-five percent of providers reported that their patients experienced side effects after using a recommended galactagogue. One provider stated, "Metoclopramide can cause a patient to feel depressed if not titrated to wean off the medication. Fenugreek causes urine to smell like maple syrup, but not a big issue, [I] just like to warn [the] patient." And a lactation consultant noted that "Overuse of fenugreek can cause nausea, and will make your body odor and urine smell like maple syrup."

Only $7.5 \%$ of respondents reported that their patients experienced adverse effects (vs side effects) after using a particular galactagogue. The only adverse effects reported were depression and fatigue for metoclopramide, which had also been reported as a side effect (Figure 3).

\section{Discussion}

Breastfeeding rates in the US are suboptimal, and women who face difficulties with breastfeeding, such as women with a high body mass index, are among the population for whom it may hold the most benefit. There is a need for greater lactation support and to avail alternative options when counseling is insufficient. This study tried to understand the perceptions, experiences, and current practice of providers regarding the recommendation of galactagogues for women with lactation difficulties not fully addressed through counseling. To date, similar investigations have not been conducted in the US and the authors are only aware of one similar study conducted among prescribers in Switzerland and Canada. ${ }^{28}$

The current study found that a large proportion of providers recommend galactagogues in their daily practice. This is in accordance with findings from Canada and Switzerland. ${ }^{28}$ By far the most popular galactagogue prescribed was fenugreek, with approximately three-quarters of prescribers indicating that they recommend fenugreek at least 'sometimes'. Other herbal remedies such as fennel, goat's rue, milk thistle, More Milk or More Milk Plus were only recommended by a small number of prescribers in this sample, perhaps due to unfamiliarity with these products, or lack of evidence on their effectiveness. Similarly, domperidone and metoclopramide were 'never' or only 'rarely' recommended. Other surveys on galactagogues have reported largely similar findings to those described here..$^{23-25,29}$ However, Swiss respondents seemed to recommend predominantly oxytocin nasal spray, herbals, and homeopathic remedies, whereas Canadian respondents used domperidone as well as herbal remedies, ${ }^{28}$ indicating differences by geographic region.

There are different dosages and forms of galactagogue preparation available ${ }^{23}$ and another recent study has illustrated that the methods of administration among users vary significantly. ${ }^{22}$ This is also reflected by the absence of evidencebased clinical guidelines to assist providers in choice and appropriate dosage of galactagogues. Consequently, in this study, about half of the respondents answered that their recommended dosage is 'as needed' without further specifying the duration of treatment or the daily doses. This is again in line with findings of the Swiss-Canadian study reporting that providers did not follow any evidence-based guideline or protocol. ${ }^{28}$ 
Due to the absence of rigorous studies on these products, including large-scale, randomized controlled trials, evidence about the efficacy of galactagogues remains lacking. Given the very small number of clinical trials conducted to date, the biological mechanisms of action of these compounds also remain largely unknown. ${ }^{25}$ In addition, most studies conducted to date have limitations regarding study design, such as poorly defined inclusion criteria, unclear sample size calculations, uncertain randomization methods, and poor reporting on adherence, making these studies prone to bias and very difficult to compare. ${ }^{25}$

Despite this lack of evidence, most prescribers reported that galactagogues were useful for their clients and would recommend galactagogues again (even in some cases where they were unsure of improvements in lactation). This perception may extend from usefulness to safety, and is likely reflective of the feedback providers get from their clients. These findings are also echoed in an Australian study which reported that lactating women using galactagogues, especially fenugreek, perceived them as useful and effective. ${ }^{22}$ The authors of that study highlighted the potential psychological benefits of herbal galactagogues in increasing confidence among lactating mothers, which benefits breastfeeding. ${ }^{30}$

The demand for galactagogues appears to be increasing, as do some risk factors for physiologic issues related to insufficient lactation, such as obesity, increasing maternal age at delivery, and caesarian section..$^{29,31,32}$ Women with physiologic issues will rely on their health provider's advice to support them. In the absence of reliable data, the use of galactagogues may be considered controversial, causing difficult choices for providers whose patients seek additional lactation support. Survey participants mentioned the absence of reliable data as the main reason for not recommending galactagogues. Domperidone, for example, has been banned in the US since $2004^{33,34}$ but is often used in the UK and Canada. ${ }^{34,35}$ For most other galactagogues, no clear guidelines are available, and in this study providers reported using a wide range of different sources to gather information, few of which may be authoritative.

\section{Limitations}

The nature of the study included convenience sampling which has inherent limitations and may have favored a selection toward prescribers who are more interested in galactagogues. This similarly applies to the fraction of non-responders. Response rates varied from question to question and low response rates can impact the level of confidence in overall results. Although participants were assured about their ano- nymity, they may have been led toward more socially desirable answers. Lastly, generalizability to other settings is difficult since providers' practices and experiences may vary in different settings (eg, urban or rural), countries, and health systems.

\section{Conclusion}

The study reported findings from a survey of providers involved in caring for lactating women and their infants. The results indicated frequent recommendation of galactagogues, as well as some concern about their use, mainly related to lack of information on safety and efficacy. The findings suggest that health providers need higher quality data from rigorous studies of galactagogues, and better clinical guidance, to provide evidence-based recommendations to breastfeeding clients. Funding and support for more robust studies, protocols, and better post-marketing pharmacovigilance systems are urgently needed to assure providers and users about the efficacy and safety of the most commonly used galactagogues.

\section{Acknowledgment}

The authors are grateful to the study respondents for their contribution.

\section{Disclosure}

The authors report no conflicts of interest in this work.

\section{References}

1. WHO. The optimal duration of exclusive breastfeeding. Report of the expert consultation. 2001. Available from: http://www.who.int/nutrition/ publications/infantfeeding/WHO_NHD_01.09/en/.

2. Innocenti declaration on the protection, promotion and support of breastfeeding. Florence, Italy; 1990. Available from: http://www.who.int/about/ agenda/health_development/events/innocenti_declaration_1990.pdf.

3. American Academy of Pediatrics. Breastfeeding and the use of human milk. Pediatrics. 2012;129(3):e827-841.

4. American College of Obstetrics and Gynecology. Breastfeeding in underserved women: increasing initiation and continuation of breastfeeding. Committee Opinion No 570. Obstet Gynecol. 2013;122: 423-428.

5. Lawrence RA. Breastfeeding, a Guide for the Medical Profession. 2nd ed. St. Louis, MO: Mosby; 1985.

6. Abrahams SW, Labbok MH. Breastfeeding and otitis media: a review of recent evidence. Curr Allergy Asthma Rep. 2011;11(6):508-512.

7. Lewallen LP, Dick MJ, Flowers J, Powell W, Zickefoose KT, Wall YG, Price ZM. Breastfeeding support and early cessation. J Obstet Gynecol Neonatal Nurs. 2006;35(2):166-172.

8. Ahluwalia IB, Morrow B, Hsia J. Why do women stop breastfeeding? Findings from the Pregnancy Risk Assessment and Monitoring System. Pediatrics. 2005;116(6):1408-1412.

9. Lawrence RA. Breastfeeding in Modern Medicine. St. Louis, MO: Mosby; 1994.

10. Renfrew MJ, McCormick FM, Wade A, Quinn B, Dowswell T. Support for healthy breastfeeding mothers with healthy term babies. Cochrane Database Syst Rev. 2012;16(5):CD001141. 
11. Chung M, Raman G, Trikalinos T, Lau J, Ip S. Interventions in primary care to promote breastfeeding: an evidence review for the U.S. Preventive Services Task Force. Ann Intern Med. 2008;149(8): 565-582.

12. Haroon S, Das JK, Salam RA, Imdad A, Bhutta ZA. Breastfeeding promotion interventions and breastfeeding practices: a systematic review. BMC Public Health. 2013;13(Suppl 3):S20.

13. Skouteris H, Nagle C, Fowler M, Kent B, Sahota P, Morris H. Interventions designed to promote exclusive breastfeeding in highincome countries: a systematic review. Breastfeed Med. 2014;9(3): 113-127.

14. Hale TW, Hartmann PE. Hale \& Hartmann's Textbook of Human Lactation. Amarillo, TX: Hale Publishing; 2007.

15. Penagos Tabares F, Bedoya Jaramillo JV, Ruiz-Cortés ZT. Pharmacological overview of galactogogues. Vet Med Int. 2014;2014:602894.

16. Campbell-Yeo ML, Allen AC, Joseph KS, Ledwidge JM, Caddell K, Allen VM, Dooley KC. Effect of domperidone on the composition of preterm human breast milk. Pediatrics. 2010;125(1):e107-114.

17. Wan EW, Davey K, Page-Sharp M, Hartmann PE, Simmer K, Ilett KF. Dose-effect study of domperidone as a galactagogue in preterm mothers with insufficient milk supply, and its transfer into milk. Br J Clin Pharmacol. 2008;66(2):283-289.

18. Hansen WF, McAndrew S, Harris K, Zimmerman MB. Metoclopramide effect on breastfeeding the preterm infant: a randomized trial. Obstet Gynecol. 2005;105(2):383-389.

19. Lewis PJ, Devenish C, Kahn C. Controlled trial of metoclopramide in the initiation of breast feeding. Br J Clin Pharmacol. 1980;9(2): 217-219.

20. Sakha K, Behbahan AG. Training for perfect breastfeeding or metoclopramide: which one can promote lactation in nursing mothers? Breastfeed Med. 2008;3(2):120-123.

21. Seema, Patwari AK, Satyanarayana L. Relactation: an effective intervention to promote exclusive breastfeeding. JTrop Pediatr. 1997;43(4): 213-216.

22. Sim TF, Hattingh HL, Sherriff J, Tee LB. The use, perceived effectiveness and safety of herbal galactagogues during breastfeeding: a qualitative study. Int J Environ Res Public Health. 2015;12(9): $11050-11071$
23. The Academy of Breastfeeding Medicine Protocol Committee. ABM clinical protocol \#9: use of galactogogues in initiating or augmenting the rate of maternal milk secretion (first revision January 2011). Breastfeed Med. 2011;6(1):41-49.

24. Zapantis A, Steinberg JG, Schilit L. Use of herbals as galactagogues. J Pharm Pract. 2012;25(2):222-231.

25. Mortel M, Mehta SD. Systematic review of the efficacy of herbal galactogogues. J Hum Lact. 2013;29(2):154-162.

26. Sim TF, Hattingh HL, Sherriff J, Tee LB. Perspectives and attitudes of breastfeeding women using herbal galactagogues during breastfeeding: a qualitative study. BMC Complement Altern Med. 2014;14:216.

27. Delvaux T, Crabbé F, Seng S, Laga M. The need for family planning and safe abortion services among women sex workers seeking STI care in Cambodia. Reprod Health Matters. 2003;11(21):88-95.

28. Winterfeld U, Meyer Y, Panchaud A, Einarson A. Management of deficient lactation in Switzerland and Canada: a survey of midwives' current practices. Breastfeed Med. 2012;7:317-318.

29. Sim TF, Sherriff J, Hattingh HL, Parsons R, Tee LBG. The use of herbal medicines during breastfeeding: a population-based survey in Western Australia. BMC Complement Altern Med. 2013;13:317.

30. Blyth R, Creedy DK, Dennis CL, Moyle W, Pratt J, De Vries SM. Effect of maternal confidence on breastfeeding duration: an application of breastfeeding self-efficacy theory. Birth. 2002;29(4):278-284.

31. Grzeskowiak LE, Lim SW, Thomas AE, Ritchie U, Gordon AL. Audit of domperidone use as a galactogogue at an Australian tertiary teaching hospital. J Hum Lact. 2013;29(1):32-37.

32. Grzeskowiak LE, Dalton JA, Fielder AL. Factors associated with domperidone use as a galactogogue at an Australian tertiary teaching hospital. J Hum Lact. 2015;31(2):249-253.

33. US Food and Drug Administration. FDA talk paper: FDA warns against women using unapproved drug, domperidone, to increase milk production. 2004 [June 7]. Available at: http://www.fda.gov/Drugs/DrugSafety/ InformationbyDrugClass/ucm173886.htm. Accessed August 4, 2015.

34. Anderson PO. The galactogogue bandwagon. J Hum Lact. 2013;29(1): $7-10$.

35. Bozzo P, Koren G, Ito S. Health Canada advisory on domperidone should I avoid prescribing domperidone to women to increase milk production [corrected]? Can Fam Physician. 2012;58(9):952-953.
Journal of Multidisciplinary Healthcare

\section{Publish your work in this journal}

The Journal of Multidisciplinary Healthcare is an international, peerreviewed open-access journal that aims to represent and publish research in healthcare areas delivered by practitioners of different disciplines. This includes studies and reviews conducted by multidisciplinary teams as well as research which evaluates the results or conduct of such teams or health

\section{Dovepress}

care processes in general. The journal covers a very wide range of areas and welcomes submissions from practitioners at all levels, from all over the world. The manuscript management system is completely online and includes a very quick and fair peer-review system. Visit http://www.dovepress.com/ testimonials.php to read real quotes from published authors. 